\title{
Inhibition of carcinogenesis by minor anutrient constituents of the diet
}

\author{
BY LEE W. WATTENBERG \\ Department of Laboratory Medicine and Pathology, University of Minnesota, Minneapolis, $M N$ \\ 55455, USA
}

Cancer can be prevented by consumption of a variety of chemical compounds. Many are naturally occurring. Others are purely synthetic. The following presentation will focus on a particular group of naturally occurring inhibitors of carcinogenesis, i.c. minor anutrient compounds. Examples of such compounds are phenols, indoles, aromatic isothiocyanates, terpenes and organosulphur compounds. Foods of plant origin frequently contain amounts of these constituents as high as several per cent. Many of the minor dietary anutrient compounds have been studied minimally because they seem to lack biological ramifications. They are not toxic unless consumed in unusually large quantities and the compounds do not appear to have nutritional requirements, at least as such requirements are currently defined. However, in recent years there has been a growing awareness that these anutrient compounds can have extremely important effects on the consequences of exposure to carcinogens, drugs and an assortment of other potentially toxic materials.

The mechanisms of action of many of the minor anutrient inhibitors of carcinogenesis are poorly understood, making it difficult to organize them into a precise pattern. One way of providing an organizational framework, is to classify inhibitors according to the time in the carcinogenic process at which they are effective. Utilizing this framework, inhibitors of carcinogenesis can be divided into three categories. The first consists of compounds that prevent the formation of carcinogens from precursor substances. In the second are compounds that inhibit carcinogenesis by preventing carcinogenic agents from reaching or reacting with critical target sites in the tissues. These inhibitors are called 'blocking agents', which is descriptive of their mechanism of action. They exert a barrier function. The third category of inhibitors acts subsequent to exposures to carcinogenic agents. These inhibitors are termed 'suppressing agents', since they act by suppressing the expression of neoplasia in cells previously exposed to doses of carcinogens that otherwise would cause cancer (Wattenberg, 1985). During the course of studies of various inhibitors it became apparent that some inhibitors act at more than one time-point in the carcinogenic process. The implication of these observations will be discussed subsequently.

The classical example of inhibition of carcinogenesis by preventing the formation of carcinogens from precursor substances entails the use of ascorbic acid to prevent formation of nitroso carcinogens from the reactions of nitrite with appropriate amines or amides. This prevention can occur in vivo, particularly in the alimentary tract (Mirvish, 1981). It also can be used as a means of preventing formation of nitroso carcinogens in food. Prevention of carcinogen formation, along with procedures for inactivating or destroying carcinogens in foods and beverages by chemical means before consumption, constitutes an extension of chemoprevention strategies into the area of environmental health. Possible examples of such strategies would include those preventing the formation of imidazoquinoxalines in meat products, those preventing the formation of urethane in alcoholic beverages, and destruction of hydrazines in mushrooms (Ough, 
1976; Miller \& Miller, 1979; Jagerstad et al. 1985; Weisburger \& Jones, 1990). An additional area of interest pertains to a group of suspect carcinogens, the fecapentaenes that are formed by the microbial flora of the large intestine of the human (Gupta et al. 1983; Baptista et al. 1984). The fecapentaenes are highly mutagenic in the Ames test and also produce a wide variety of other genotoxic reactions. If the fecapentaenes prove to be full or partial carcinogens in the human, strategies to prevent their synthesis or to inactivate them could become a component of an overall programme for preventing cancer of the large bowel.

\section{BLOCKING AGENTS}

Mechanisms of action. A major portion of this presentation will focus on the inhibitory effects of blocking agents. Blocking agents prevent carcinogens from reaching or reacting with critical target sites by several mechanisms. One of these is to inhibit activation reactions of carcinogens requiring metabolic activation. A second entails induction of increases in the activities of enzyme systems that can detoxify carcinogens. A third mechanism is the trapping of reactive carcinogenic species. Each of these will be discussed in turn.

Blocking agents that act by inhibiting carcinogen activation. Several groups of chemopreventive agents prevent carcinogenesis by inhibiting activation reactions of carcinogens. Such activation reactions are required to produce the reactive chemical species that attack critical target sites. An early example of a chemopreventive agent that acts by this mechanism is disulfiram. Disulfiram is a potent inhibitor of 1,2dimethylhydrazine(DMH)-induced carcinogenesis of the large bowel in rodents. DMH undergoes a series of metabolic reactions that result in the formation of the ultimate carcinogenic species, a highly reactive electrophile. Disulfiram has been found to block this sequence of activation reactions at two steps. The elucidation of these enzyme inhibitions has been the result of extensive investigations by Fiala et al. (1977). In experimental animals administration of disulfiram before and during the course of administration of DMH prevents large bowel carcinogenesis (Wattenberg, 1975). Disulfiram is a compound with substantial toxicity so that its use as a chemopreventive agent in the human is not practical.

More recently, several groups of naturally occurring compounds also have been found to prevent carcinogenesis by inhibiting carcinogen-activation reactions (Table 1). Since almost all carcinogens occurring in food require metabolic activation, blocking agents inhibiting these reactions could play an important role in preventing neoplasia resulting from this type of exposure. Benzyl isothiocyanate is one such naturally occurring blocking agent. Benzyl isothiocyanate is found in cruciferous vegetables. It is formed by the hydrolysis of the glucosinolate, glucotropaeolin. The glucosinolate is the storage form of the compound in plant material. When the plant is processed or damaged, the glucosinolate undergoes hydrolysis with resultant formation of the free aromatic isothiocyanate (Fenwick et al. 1983). In early experiments benzyl isothiocyanate was found to inhibit 7,12-dimethylbenz(a)anthracene(DMBA)-induced mammary tumour formation in rats when given by oral intubation $2 \mathrm{~h}$ before the carcinogen (Wattenberg, 1977). In more recent work, the effects of benzyl isothiocyanate on nitrosamine metabolism in rat liver microsomes and cultured oesophagus was studied by Chung $e t$ al. $(1984,1985)$. These investigators found that administration of benzyl isothiocyanate $2 \mathrm{~h}$ 


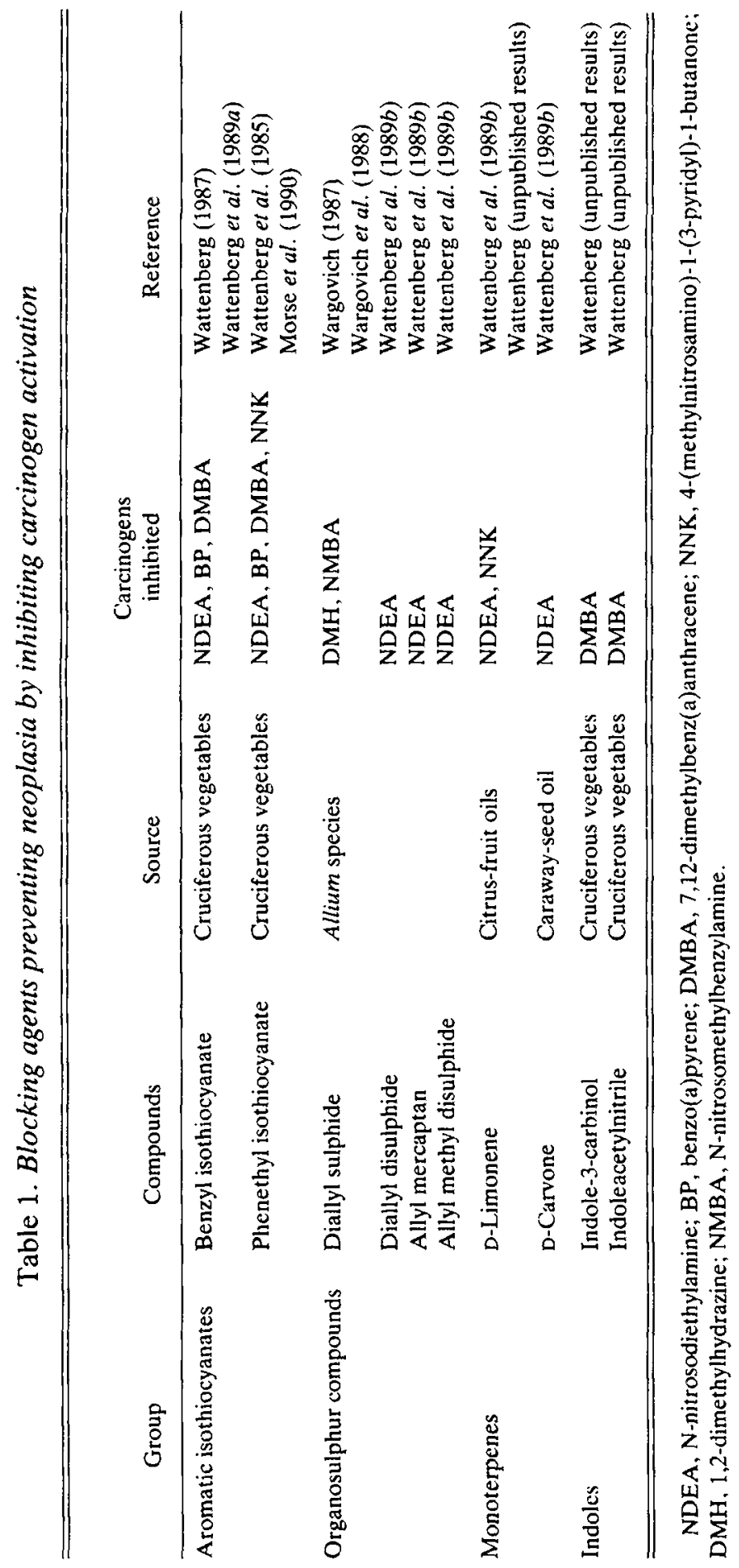


Table 2. Effects of test compounds on induction of neoplasia by the tobacco-specific carcinogen 4-(methylnitrosamino)-1-(3-pyridyl)-1-butanone (NNK) in female A/J mice

(Mean values with their standard errors. Cottonseed oil $(0.2 \mathrm{ml})$ or the test matcrial in $0.2 \mathrm{ml}$ cottonseed oil were given by oral intubation $1 \mathrm{~h}$ before $0.5 \mathrm{mg} \mathrm{NNK}$ in $0.1 \mathrm{ml}$ tricaprylin. The first sequence of administrations was given to female $\mathrm{A} / \mathrm{J}$ mice when they were 9 weeks of age. The administrations were repcated twice weekly for 8 weeks. The experiment was terminated 28 weeks after the initial dose of NNK)

\begin{tabular}{|c|c|c|c|c|c|c|c|c|}
\hline \multirow[b]{3}{*}{ Pretreatment } & \multirow{3}{*}{$\begin{array}{l}\text { No. of } \\
\text { mice at } \\
\text { riskt }\end{array}$} & \multicolumn{4}{|c|}{ Forestomach tumours } & \multirow{2}{*}{\multicolumn{2}{|c|}{$\begin{array}{c}\text { Pulmonary } \\
\text { adenomas } \\
\text { (no. of } \\
\text { tumours } \\
\text { per mouse) } \ddagger\end{array}$}} & \multirow{3}{*}{$\begin{array}{l}\text { Wt gain } \\
\text { from } 8 \text { to } \\
37 \text { weeks } \\
\text { of age }(\mathrm{g})\end{array}$} \\
\hline & & \multirow{2}{*}{$\begin{array}{c}\text { Percentage } \\
\text { of mice } \\
\text { with } \\
\text { papillomas }\end{array}$} & \multicolumn{2}{|c|}{$\begin{array}{l}\text { No. of } \\
\text { papillomas } \\
\text { per mouse }\end{array}$} & \multirow{2}{*}{$\begin{array}{l}\text { Percentage } \\
\text { of mice } \\
\text { with } \\
\text { carcinomas }\end{array}$} & & & \\
\hline & & & Mean & SF: & & Mean & $\mathrm{SF}$ & \\
\hline \multicolumn{9}{|l|}{ Vehicle } \\
\hline (cottonseed oil) & 20 & 90 & $2 \cdot 8$ & 0.6 & 5 & $50 \cdot 8$ & $2 \cdot 6$ & $4 \cdot 6$ \\
\hline \multicolumn{9}{|l|}{ Dipropyldisulphide } \\
\hline$(2 \cdot 5 \mathrm{mg})$ & 15 & $7^{*}$ & $0.07 *$ & 0.07 & 0 & $57 \cdot 0$ & 1.6 & $5 \cdot 4$ \\
\hline D-Limonene $(2.5 \mathrm{mg})$ & 15 & $0^{*}$ & \multicolumn{2}{|c|}{$0^{*}$} & 0 & $15 \cdot 3 *$ & $2 \cdot 1$ & $4 \cdot 0$ \\
\hline
\end{tabular}

* Mean values for the test compound were significantly different from those of the vehicle: $P<0.001$.

+ Mice surviving the duration of the protocol.

$\ddagger$ No. of tumours in the entire group/number of mice at risk.

before death markedly inhibited activation of $\mathrm{N}$-nitrosodimethylamine (NDMA) and the tobacco-specific carcinogen, 4-(methylnitrosamino)-1-(3-pyridyl)-1-butanone (NNK). Subsequently, benzyl isothiocyanate administered orally $15 \mathrm{~min}$ before oral carcinogen challenge was found to inhibit the neoplastic effects of diethylnitrosamine (NDEA) and benzo(a)pyrene (BP) (Wattenberg, 1987).

Organosulphur compounds found in Allium species, including garlic, onions, leeks and shallots (Block, 1985; Fenwick \& Hanley, 1985a,b.c) are a second group of naturally occurring compounds that also have been found to inhibit carcinogenesis by preventing carcinogen activation. In one series of experiments, they inhibited NDEA-induced carcinogenesis of the forestomach and, to a lesser extent, the lungs in female $\mathrm{A} / \mathrm{J}$ mice (Wattenberg et $a l$. $1989 a, b$ ). The most potent of the naturally occurring organosulphur compounds in this experimental model was diallyl disulphide. In other studies Wargovich et al. (1988) have shown that diallyl sulphide inhibits $\mathrm{N}$-nitrosomethylbenzylamineinduced oesophageal cancer in rats when the organosulphur compound was administered orally $3 \mathrm{~h}$ before the nitrosamine. In related work, Brady et al. (1988) have demonstrated that diallyl sulphide inhibits the microsomal metabolism of nitrosamines when administered $3 \mathrm{~h}$ before death of rats. Inhibition of DMH-induced neoplasia of the large bowel in mice has also been shown to be brought about by diallyl sulphide when this compound was administered orally $3 \mathrm{~h}$ before the carcinogen (Wargovich, 1987). In recent work dipropyl disulphide showed some inhibition against NNK (Table 2).

A third group of naturally occurring compounds that inhibit carcinogen activation and carcinogenesis when given shortly before carcinogen exposure are monoterpenes. Two such compounds have been investigated, D-limonene and D-carvone. D-Limonene is a major constituent of citrus-fruit oils (Shaw, 1979). For example, orange oil contains over 
90\% D-limonene. D-Carvone is a major constituent of caraway-seed oil. This oil contains approximately 50\% D-carvone (Solzin, 1977). D-Limonene, D-carvone, orange oil and caraway-seed oil all have been found to inhibit activation of NDMA (L. W. Wattenberg and J. L. Leong, unpublished results). Carcinogenesis experiments have been carried out in which D-limonene, D-carvone, or caraway-seed oil were administered orally to female A/J mice $1 \mathrm{~h}$ before NDEA (Wattenberg et al. 1989a,b). All three substances had a profound inhibitory effect on forestomach tumour formation and also inhibited the occurrence of pulmonary adenomas in these animals. More recently it has been found that D-limonene will inhibit the carcinogenicity of NNK (Table 2).

In additional studies, glucobrassicin was found to inhibit DMBA-induced mammary neoplasia in rats when administered orally $4 \mathrm{~h}$ before the carcinogen. Preliminary findings also show that indole-3-carbinol and, to a lesser extent, indoleacetylnitrile inhibit mammary tumour formation under comparable conditions (L. W. Wattenberg, unpublished results). Thus, information is now available that four widely occurring groups of naturally occurring compounds, i.e. aromatic isothiocyanates, organosulphur compounds found in Allium species, monoterpenes and indoles, have the capacity to inhibit carcinogenesis when administered shortly before carcinogen challenge.

Blocking agents that act by increasing carcinogen detoxification. Blocking agents can prevent the occurrence of neoplasia by increasing the detoxification of carcinogens. Two general categories of blocking agents acting by this mechanism have been identified. They are designated type A and type B inhibitors (Wattenberg, 1985). Type A inhibitors induce an increase in phase 2 enzymes, i.e. conjugating enzymes and some related protective systems. A prominent feature of these enzyme inductions is a marked increase in glutathione S-tranferase ( $E C 2.5 .1 .18$ ) activity. UDP-glucuronosyl transferase activity is also enhanced, as are epoxide hydrolase $(E C$ 3.3.2.3) and $\mathrm{NAD}(\mathrm{P}) \mathrm{H}$-quinone reductase $(\mathrm{NAD}(\mathrm{P}) \mathrm{H}$ : (quinone-acceptor) oxidoreductase; $E C$ 1.6.99.2) activities. Another feature of type $A$ inhibitors is an alteration in microsomal metabolism in which there is little change in overall activity. However, a pronounced alteration in metabolite pattern occurs as demonstrated with benzo(a)pyrene (BP) as a substrate. In addition to enzymic changes, an increase in the levels of glutathione in tissues is found. Type $B$ inhibitors enhance both phase 1 and phase 2 enzyme activities. Reviews of the effects of type A and type B inhibitors have been published previously (Wattenberg, 1985).

Since type A inhibitors have been found to be highly effective, a continuing effort has been made to identify compounds belonging to this category of chemopreventive agents. Recently, work of this nature has focused on the organosulphur compounds found in Allium species. In initial studies, the capacity of allyl methyl trisulphide (AMT) to induce increased glutathione S-transferase activity in rodent tissues was investigated. AMT induces increased activity of this enzyme system when given 4 and $2 \mathrm{~d}$ before assay. Using this administration schedule, AMT then was found to inhibit BP-induced neoplasia of the forestomach of female A/J mice (Sparnins et al. 1986). Experimental protocols in which the test compound is given 96 and $48 \mathrm{~h}$ before carcinogen challenge are commonly used for studying the inhibitory effects of agents that act by inducing increased activity of detoxification systems.

In subsequent work, additional organosulphur compounds were studied under these conditions. Ultimately, the experiments have included four allyl group-containing compounds with one, two, or three linearly-connected sulphur atoms: AMT, allyl methyl 
disulphide (AMD), diallyl trisulphide (DAT), and diallyl sulphide (DAS). Four corresponding saturated compounds in which propyl groups have been substituted for the allyl groups also were studied. All four allylic compounds inhibited BP-induced neoplasia of the forestomach. Their saturated analogues were almost without inhibitory activity, indicating the importance of the allyl groups. DAT, which contains two allyl groups, is more potent than AMT, which contains only one allyl group, thus providing further evidence for the role of allyl groups in the inhibitory effects observed. DAS and AMD, but not DAT or AMT, inhibited pulmonary adenoma formation (Sparnins et al. 1988). The fact that in the lung the monosulphide and disulphide inhibit, but the trisulphide does not inhibit, indicates that the number of sulphur atoms in the molecule can control the organ sites at which protection against carcinogenesis will occur. All four allylic compounds induce increased glutathione S-transferase activity in the forestomach, but vary in their capacity to induce glutathione S-transferase activity in the lung, liver and small bowel. Their saturated analogues produced little or no induction (Sparnins et al. 1988). Like the organosulphur compounds from Allium species, aromatic isothiocyanates from cruciferous vegetables, in particular, benzyl isothiocyanate, induce increased glutathione S-transferase activity and have other properties of type A inhibitors.

\section{SUPPRESSING AGENTS}

Suppressing agents have been found in cruciferous vegetables and also in orange oil (Wattenberg, 1983). In the initial work with cruciferous vegetables, dehydrated powders prepared from Chieftain Savoy cabbage were shown to inhibit DMBA-induced mammary tumour formation. In further investigations, it was found that frozen-thawed segments of cabbage leaf added directly to cages of rats produced a similar level of reduction of neoplasia (Wattenberg et al. 1989a,b). The amount of cabbage leaf fed was of the order of $5 \mathrm{~g} / \mathrm{kg}$ body-weight per d. More recently, similar studies have been done with frozen-thawed segments of broccoli. In these experiments in which the suppressive capacities of frozen-thawed cabbage and broccoli have been investigated, the cabbage (Chieftain Savoy) or broccoli (Premium Crop) were given beginning 1 week after the DMBA and were continued for the duration of the experiments (18 weeks after DMBA). The rats were maintained on a semi-purified diet. The vegetable additions were given $6 \mathrm{~d} /$ week. The results of these experiments showed that fewer animals receiving cabbage had mammary tumours, as compared to those not receiving the supplement $(100 \% v .56 \%)$ and the average number of tumours per rat was diminished markedly $(2.5$ v. 0.8). Similar findings were obtained with broccoli additions (Wattenberg et al. 1989a). Inhibition was obtained by feeding either the floret or stem. In an additional experiment with broccoli, it was found that if the vegetable supplement was provided for only 5 weeks, i.e. from 1 week to 6 weeks after carcinogen administration, a significant inhibition of mammary neoplasia was obtained.

Possibly related to the suppressive effects of cruciferous vegetables has been the finding that benzyl isothiocyanate, a constituent of these plants, will inhibit DMBAinduced mammary carcinogenesis when administered beginning 1 week after challenge by DMBA (Wattenberg, 1981). In other work, suppressive effects have been produced by administration of orange oil or its principal constituent, D-limonene (Wattenberg, 1981, 1983; Elson et al. 1988). The observations that suppressing agents occur in 
commonly consumed dietary constituents indicate that further searches should be made for this category of chemopreventive agent.

\section{INHIBITORS WITH BOTH BLOCKING AND SUPPRESSING ACTIVITY}

During the course of studies of inhibitors of carcinogenesis, it has become apparent that some substances have both blocking and suppressing effects. The first such compound that we studied was sodium cyanate. This compound has both blocking and suppressing effects against DMBA-induced mammary carcinogenesis in the rat (Wattenberg, 1980, 1981). In later work, cruciferous vegetables were shown to have these characteristics, as did one of their constituents, i.e. benzyl isothiocyanate. In the studies with cabbage, consumption of a semi-purified diet to which dehydrated powders prepared from Chieftain Savoy cabbages had been added, produced blocking effects against DMBAinduced mammary carcinogenesis in the rat. In addition, it was found that consumption of the cabbage powders beginning 1 week after administration of DMBA resulted in suppression of mammary tumour formation. In subsequent work, benzyl isothiocyanate was shown to have a blocking effect against DMBA-induced mammary carcinogenesis and also to suppress formation of mammary tumours under similar conditions to those used with crude plant materials (Wattenberg, 1981).

In other work, citrus-fruit oils have been found to have both blocking and suppressing capacities. The most extensively studied of these oils has been orange oil. This oil has a blocking effect against DMBA-induced mammary neoplasia and also exerts a suppressive effect in this experimental model (Wattenberg, 1983). In experiments with constituents of orange oil, D-limonene has been shown to have both blocking and suppressing effects against DMBA-induced mammary tumour formation (Elson et al. 1988). In addition to inhibition of neoplasia, cruciferous vegetable powders, benzyl isothiocyanate, orange oil, and D-limonene have another property in common, i.e. they all induce increased glutathione S-transferase activity in mouse tissues. Comparable studies have not yet been reported for the rat.

The finding of inhibitors having both blocking and suppressing activity would appear to be important. However, the mechanism or mechanisms which result in these dual inhibitory actions is uncertain. Two distinctive possibilities merit consideration, although others also may exist. One is that a coordinated pleiotropic protective system against potentially toxic compounds exists that entails both a detoxification component and a suppressive component. The latter would be a 'fail-safe' means of protection in the event that the former was not completely effective. On exposure to toxic compounds, the detoxification systems constitute the first line of defence. Phase 2 enzymes, as well as glutathione, are components of this detoxification (blocking) defence. Since blocking effects might not always be completely successful, a second line of defence would be required that would entail preventing the manifestations of any damage that occurred, i.e., 'suppression'.

Whereas a coordinated protective mechanism entailing increased activity of detoxification systems and suppression of manifestations of toxicity would appear to be a reasonable possibility, the inclusion into such a coordinated mechanism of a decrease in activities of cytochrome P-450 enzymes might appear incongruous. However, studies by Deloria et al. (1985) and Gooderham \& Mannering (1986) offer some insight as to how such enzyme inhibitions might fit into an overall protective system. These investigators 
have shown that some compounds that reduce cytochrome P-450 activity increase interferon levels in tissues. Such findings suggest that a decrease in activity of one mechanism that is involved in protective events can be accompanied by an enhanced activity of a different protective system. The inclusion of DNA repair systems as a component of suppressive mechanisms also is a consideration. Additional mechanisms may exist for preventing manifestations of damage to other cellular constituents. If a coordinated defence mechanism with both blocking and suppressing components exists, it is possible that it is triggered by a receptor mechanism. This certainly would be a point for future investigation.

A second possibility that could account for a compound having both blocking and suppressing activity would be that the chemical is highly reactive and simply has multiple effects due to this attribute. If such is the case, then the blocking and suppressing effects would be triggered separately. Aromatic isothiocyanates are highly reactive compounds and their capacity to act as both blocking and suppressing agents might be accounted for on the basis of this high chemical reactivity. In contrast, D-limonene is not a highly reactive molecule. However, it is metabolized to epoxides which do have high reactivity. At the present time, the paucity of information and the characteristics of the inhibitors make it difficult to evaluate the two major mechanisms of action considered. The identification of a receptor activating both blocking and suppressing action, if such occurs, would be a major achievement. But even if such a unified defence system does not exist, a knowledge of the characteristics of inhibitors having both blocking and suppressing effects might enhance our information base for obtaining chemopreventive agents with maximum protective capacities.

\section{INTERIM CLASSIFICATION OF FOODS IN TERMS OF THEIR POTENTIAL IMPACT ON RISK OF NEOPLASIA}

Foods are very complicated in that they contain very large numbers of chemical compounds. The compounds can include those that have protective effects against carcinogenesis or, on the other hand, chemicals that can cause or contribute to a neoplastic response. Although our current state of knowledge is very incomplete, a simple interim organizational scheme might be helpful. The scheme would place foods into four categories: (1) protective, i.e., those foods containing substantial quantities of protective compounds and, at most, trivial amounts of carcinogenic agents; (2) mixed, i.e., those foods containing substantial quantities of both protective substances and compounds with cancer-causing attributes; (3) with risk, i.e., those foods containing substantial quantities of compounds that can cause or contribute to a neoplastic process and, at most, trivial amounts of protective chemicals, and (4) inactive, i.e., foods that contain, at most, trivial amounts of both protective substances and compounds with cancer-causing attributes (Table 3 ). This scheme obviously is severely compromised by a paucity of information. However, even with this difficulty, it would have usefulness in providing a framework into which existing and future information can be placed. In addition, such a scheme would also be helpful in directing attention at means of moving towards the objective of achieving a diet with optimal protective capacities against the occurrence of cancer. 
Table 3. Interim classification of foods in terms of their potential impact on risk of neoplasia

\begin{tabular}{|c|c|c|c|}
\hline $\begin{array}{l}\text { Food } \\
\text { designation }\end{array}$ & $\begin{array}{l}\text { Content of } \\
\text { substances } \\
\text { protecting } \\
\text { against } \\
\text { cancer }\end{array}$ & $\begin{array}{l}\text { Content of } \\
\text { substances } \\
\text { enhancing } \\
\text { risk of } \\
\text { cancer }\end{array}$ & Future objectives \\
\hline Protective & Substantial & Trivial or none & $\begin{array}{l}\text { This is an ultimate goal } \\
\text { Validation must be extremely thorough }\end{array}$ \\
\hline Mixed & Substantial & Substantial & $\begin{array}{l}\text { Selective breeding, processing, or genetic } \\
\text { engineering to decrease or eliminate risk- } \\
\text { enhancing compounds and if possible, } \\
\text { increase content of protective compounds }\end{array}$ \\
\hline With risk & Trivial or none & Substantial & $\begin{array}{l}\text { Selective breeding, processing, or genetic } \\
\text { engineering to decrease or eliminate risk- } \\
\text { enhancing compounds }\end{array}$ \\
\hline Inactive & Trivial or none & Trivial or none & Validification important \\
\hline
\end{tabular}

\section{SUMMARY}

A continuing study of chemopreventive agents has focused on several categories of naturally occurring compounds that inhibit carcinogen activation and are effective in preventing carcinogen-induced neoplasia when administered at short time-intervals before carcinogen challenge. The inhibitory compounds are: aromatic isothiocyanates found in cruciferous vegetables, monoterpenes present in citrus fruits and caraway-seed oil, and organosulphur compounds occurring in Allium species. Preliminary work indicates that glucobrassicin and indoles existing in crucifcrous vegetables also have these attributes. Almost all carcinogens that are consumed in food require metabolic activation. Thus, inhibition of carcinogen activation reactions could be effective against this type of exposure. In addition, three naturally occurring compounds, i.e. phenethyl isothiocyanate, D-limonene and dipropyl sulphide inhibit activation of the tobaccospecific carcinogen NNK, and accordingly may have the capacity to diminish carcinogenic response to exposures to tobacco.

The property of cruciferous vegetables, orange oil, benzyl isothiocyanate, and $\mathrm{D}$-limonene, to act as both blocking and suppressing agents has been discussed. Two possible mechanisms for this multi-phase activity were presented. The first is that these inhibitory substances activate a complex integrated defence mechanism against toxic compounds which entails both blocking and suppressing components. The blocking component is the initial line of defence, and the suppressing component constitutes a 'fail-safe' backup to assure that if any of the toxic material attacks cellular constituents, its effects will be nullified. The second possible mechanism considered is that the inhibitors, because of high reactivity, have multiple biological effects that are separate and not part of a single, coordinated response. Inhibitors that have both blocking and suppressing effects could be particularly useful as chemopreventive agents.

A simple interim classification of foods in terms of their potential impact on the occurrences of cancer has been proposed. 


\section{REFERENCES}

Baptista, J., Bruce, W. R., Gupta, I., Krepinsky, J. J., Van Tassell, R. L. \& Wilkins, T. D. (1984). On distribution of different fecapentaenes, the fecal mutagens, in the human population. Cancer Letters 22 , 299-303.

Block, E. (1985). The chemistry of garlic and onions. Scientific American 252, 114-119.

Brady, J. F., Li, D., Ishizaki, H. \& Yang. C. S. (1988). Effect of diallyl sulfide on rat liver microsomal metabolism and other monooxygenase activities. Cancer Research 48, 5937-5940.

Chung, F. L., Juchatz, A. \& Hecht, S. S. (1984). Effects of dietary compounds on $\alpha$-hydroxylation of $N$-nitrosopyrrolidine and $N^{\prime}$-nitrosonornicotine in rat target tissues. Cancer Research 44, 2924-2928.

Chung, F. L., Wang, M. \& Hecht, S. S. (1985). Effects of dietary indoles and isothiocyanates on $N$-nitrosodimethylamine and 4-(methylnitrosamine)-1-(3-pyridyl)-1-butanone $\alpha$-hydroxylation and DNA methylation in rat liver. Cancer Research 45, 539-543.

Deloria, L., Abbott, V., Gooderham, N. \& Mannering, G. J. (1985). Induction of xanthine oxidase and depression of cytochrome P-450 by interferon inducers: genetic differences in the responses of mice. Biochemical and Biophysical Research Communications 131, 109-114.

Elson, C. E., Maltzman. T. H., Boston, J. L. \& Tabber, M. A. (1988). Anti-carcinogenic activity of D-limonene during initiation and promotion/progression stages of DMBA-induced mammary carcinogenesis. Carcinogenesis $9,331-332$.

Fenwick, G. R. \& Hanley, A. B. (1985a). The genus Allium. CRC Critical Revues in Food Science and Nutrition 22, 199-271.

Fenwick, G. R. \& Hanley, A. B. (1985b). The genus Allium. Part 2. CRC Critical Revues in Food Science and Nutrition 22, 273-377.

Fenwick, G. R. \& Hanley, A. B. (1985c). The genus Allium. Part 3. CRC Critical Revues in Food Science and Nutrition 23, 1-73.

Fenwick, G. R., Heaney, R. K. \& Mullin, W. J. (1983). Glucosinolates and their breakdown products in food and food plants. CRC Critical Revues in Food Science and Nutrition 18, 123-201.

Fiala, E. S., Bobotas, G., Kulakis, C., Wattenberg, L. W. \& Weisburger, J. (1977). The effects of disulfiram and related compounds on the in vivo metabolism of the colon carcinogen 1,2-dimethylhydrazine. Biochemical Pharmacology 26, 1763-1768.

Gooderham, N. J. \& Mannering, G. J. (1986). Depression of cytochrome P-450 and alterations of protein metabolism in mice treated with the interferon inducer polyriboinosinic acid, polyribocytidylic acid. Archives of Biochemistry and Biophysics 250, 418-425.

Gupta, I., Baptista, J., Bruce, W. R., Che, C. T., Furrer, R., Gingerich, J. S., Grey, A. A., Marai, L., Yates, P. \& Krepinsky, J. J. (1983). Structures of fecapentaenes, the mutagens of bacterial origin isolated from human feces. Biochemistry 22, 241-245.

Jagerstad, M., Reutersward, A. L., Griuas, S., Olson, K., Neigishi, C. \& Sato, S. (1985). Effects of meat composition and cooking conditions on the formation of mutagenic imidazoquinoxalines. In Diet, Nutrition and Cancer, pp. 87-96 [Y. Hayashi, M. Nagao, T. Sugimura, S. Takayama, L. Tomatis, L. Wattenberg and G. Wogan, editors]. Tokyo: Japan Scientific Societies Press.

Miller, E. C. \& Miller, J. A. (1979). Naturally-occurring chemical carcinogens that may be present in foods. International Revue of Biochemistry 27, 123-165.

Mirvish, S. S. (1981). Inhibition of the formation of carcinogenic $N$-nitroso compounds by ascorbic acid and other compounds. In Cancer Achievements, Challenges and Prospects for the 1980's, pp. 557-588 [J. H. Burchenal and H. F. Oettgen, editors]. New York: Gruen and Stratton.

Morse, M. A., Hecht, S. S. \& Chung, F. L. (1990). Inhibition of tobacco-specific nitrosamine 4(methylnitrosamino)-1-(3-pyridyl)-1-butanone (NNK)-induced lung tumors and DNA methylation in F344 rats and $\mathrm{A} / \mathbf{J}$ mice by phenethyl isothiocyanate. In Antimutagenesis and Anticarcinogenesis Mechanisms II, pp. 345-350 [Y. Kuroda, D. M. Shankel and M. D. Waters, editors]. New York: Plenum Press.

Ough, C. S. (1976). Ethyl carbamate in fermented beverages and foods. 1. Naturally-occurring ethyl carbamate. Journal of Agricultural and Food Chemistry 24, 323-331.

Shaw, P. E. (1979). Review of quantitative analyses of citrus essential oils. Agricultural Food Chemistry 27, 246-257.

Solzin, U. J. (1977). The analysis of essential oils and extracts (oleoresins) from seasonings - a critical review. CRC Critical Revues in Food Science and Nutrition 345, 373.

Sparnins, V. L., Barany, G. \& Wattenberg, L. W. (1988). Effects of organosulfur compounds from garlic and onions on benzo(a)pyrene-induced neoplasia and glutathione S-transferase activity. Carcinogenesis 9 , $131-134$. 
Sparnins, V. L., Mott, A. W., Barany, G. \& Wattenberg, L. W. (1986). Effects of allyl methyl trisulfide on glutathione S-transferase activity and BP-induced neoplasia in the mouse. Nutrition and Cancer 8, 211-215.

Wargovich, M. J. (1987). Diallyl sulfides, a flavor component of garlic (Allium sativum) inhibits dimethylhydrazine-induced colon cancer. Carcinogenesis 8, 487-489.

Wargovich, M. J., Woods, C., Eng, V. W. S., Stephens, L. C. \& Gray, K. (1988). Chemoprevention of $N$-nitrosomethylbenzylamine-induced esophageal cancer in rats by the naturally-occurring thioether, diallyl sulfide. Cancer Research 48, 6872-6875.

Wattenberg, L. W. (1975). Inhibition of dimethylhydrazine-induced neoplasia of the large intestine by disulfiram. Journal of the National Cancer Institute 54, 1005-1006.

Wattenberg, L. W. (1977). Inhibition of carcinogenic effects of polycyclic hydrocarbons by benzyl isothiocyanate and related compounds. Journal of the National Cancer Institute 58, 395-398.

Wattenberg, L. W. (1980). Inhibition of polycyclic hydrocarbon-induced neoplasia by sodium cyanate. Cancer Research 40, 232-234.

Wattenberg, L. W. (1981). Inhibition of carcinogen-induced neoplasia by sodium cyanate, tert-butylisocyanate and benzyl isothiocyanate administered subsequent to carcinogen exposure. Cancer Research 41, 29912994.

Wattenberg, L. W. (1983). Inhibition of neoplasia by minor dietary constituents. Cancer Research 43, 2448S-2453S.

Wattenberg, L. W. (1985). Chemoprevention of cancer. Cancer Research 45, 1-8.

Wattenberg, L. W. (1987). Inhibitory effects of benzyl isothiocyanate administration shortly before diethylnitrosamine or benzo(a)pyrene on pulmonary forestomach neoplasia in $\mathrm{A} / \mathrm{J}$ mice. Carcinogenesis 8 , 1971-1973.

Wattenberg, L. W., Hanley, A. B., Barany, G., Sparnins, V. L., Lam, L. K. \& Fenwick, G. R. (1985). Inhibition of carcinogenesis by some minor dietary constituents. In Diet, Nutrition and Cancer, pp. 193-203 [Y. Hayashi, M. Nagao, T. Sugimura, S. Takayama, L. Tomatis, L. Wattenberg and G. Wogan, editors]. Tokyo: Japan Scientific Societies Press.

Wattenberg, L. W., Schafer, H. W., Waters, L. \& Davis, D. W. (1989a). Inhibition of mammary tumor formation by broccoli and cabbage. Proceedings of the American Association for Cancer Research $\mathbf{3 0}, 181$.

Wattenberg, L. W., Sparnins, V. L. \& Barany, G. (1989h). Inhibition of $N$-nitrosodiethylamine carcinogenesis by naturally-occurring organosulfur compounds and monoterpenes. Cancer Research 49, $2689-2692$.

Weisburger, J. H. \& Jones, R. C. (1990). Prevention of formation of important mutagens/carcinogens in the human food chain. In Antimutagenesis and Anticarcinogenesis Mechanisms II, pp. 105-118 [Y. Kuroda, D. M. Shankel and M. D. Waters, editors]. New York: Plenum Press. 